\title{
Hardware injection of simulated continuous gravitational wave signals for GEO 600
}

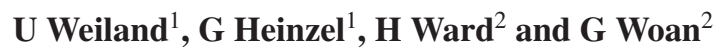 \\ ${ }^{1}$ Max-Planck-Institute for Gravitational Physics (Albert-Einstein-Institute) and University of \\ Hannover, Am kleinen Felde 30, 30167 Hannover, Germany \\ 2 Department of Physics and Astronomy, University of Glasgow, Glasgow G12 8QQ, UK \\ E-mail: uta.weiland@aei.mpg.de
}

Received 1 September 2003

Published 10 February 2004

Online at stacks.iop.org/CQG/21/S861 (DOI: 10.1088/0264-9381/21/5/073)

\begin{abstract}
Search algorithms for continuous gravitational waves are best tested by injecting simulated signals into the detector hardware. These signals need high phase and amplitude stability for reliable calibration. At GEO 600 we have developed an instrument to inject a simulated pulsar signal with a phase error less than $1 \%$ of $2 \pi$ from the correct signal phase. The injection can run over several months and can recover from interruptions. A key component of the instrument is an analogue electronic signal that can be applied to an actuator of one of the test masses of the interferometer. For the generation of this signal a microcontroller is operated as a direct digital frequency synthesiser (DDS). A digital phase locked loop (PLL) on a control computer controls the phase accumulator of the microcontroller via its phase increment register. The signal to be injected is calculated within the LIGO/LSC algorithm library on the control computer.
\end{abstract}

PACS number: $04.80 . \mathrm{Nn}$

\section{Introduction}

A new upper limit on the strength of a pulsar's gravitational wave emission was set [1] during the first observational science run, named S1, of laser interferometric gravitational wave detectors from 23 August to 9 September 2002 [2]. The most realistic test of the search algorithms used to set this limit can be made with the help of hardware injected simulated continuous gravitational wave signals, with high accuracy in phase and amplitude over long periods of time. Our set-up for the hardware injection aims for a phase error of less than $1 \%$ of $2 \pi$ from the correct signal phase and an amplitude error below $1 \%$ for injection times of several months and signal frequencies between $50 \mathrm{~Hz}$ and $2 \mathrm{kHz}$. A phase error of $1 \%$ will not affect the sensitivity of the search algorithms, and an amplitude accuracy of $1 \%$ is better than the accuracy of the injection hardware [3]. 
To inject a strain signal into the interferometer an electronic signal proportional to the gravitational wave amplitude is used to produce a force that acts upon a mirror of one of the interferometer arms, thereby changing the differential length of the two arms and simulating the effect of a gravitational wave. In GEO 600 the force can be produced by a coil-magnet pair, an electrostatic drive or a photon drive [3,4]. As the resonance frequencies of the mirror, suspended as a triple pendulum, are around $1 \mathrm{~Hz}$ and the pendulum has a high Q [5], the phase of the transfer function from force application to mirror displacement is flat in the relevant frequency region. The amplitude of this transfer function is proportional to $1 / f^{4}$ for the coil-magnet pair. With an annual Doppler shift of the signal frequency $f_{0}$ of approximately $\pm \Delta f / f_{0}=2 \times 10^{-4}$ this leads to an amplitude error of $\pm 0.08 \%$ and can thus be neglected.

Splitting the continuous gravitational wave signal into a slowly varying amplitude component and a sinusoidal phase component, as described below, allows us to generate these two components independently of each other. We use a Hitachi $3048 \mathrm{~F}$ microcontroller (running at $16 \mathrm{MHz}$ ) with two on-chip D/A converters as a direct digital frequency synthesiser (DDS) to generate the signal. The sinusoidal component and the slowly varying amplitude factor are separately generated by the two D/A converters. The parameters for the DDS are set by a control computer (an office-type PC running Linux) that is connected through an optically isolated serial link (RS-232) to the microcontroller. The phase accumulator of the microcontroller is phase locked to the desired signal phase by a digital phase locked loop (PLL) running on the control computer.

\section{The signal}

The continuous gravitational wave signal from a non-axisymmetric non-precessing spinning neutron star (i.e., with a 'wobble angle' of $\pi / 2$ [6]) produces a strain

$$
h(t)=F_{+}(t, \psi) \frac{1}{2} h_{0}\left(1+\cos ^{2} \iota\right) \cdot \cos 2 \phi(t)+F_{\times}(t, \psi) h_{0} \cos \iota \cdot \sin 2 \phi(t)
$$

at a detector on earth [6]. Here $h_{0}$ is the strain amplitude, $\iota$ the angle between the neutron star's spin direction and the propagation direction of the waves, $\phi(t)$ the phase evolution of the Doppler shifted star's spin; $F_{+}(t, \psi)$ and $F_{\times}(t, \psi)$ the antenna response of the detector to the plus and cross polarizations of the gravitational wave, respectively, and $\psi$ is the polarization angle of the wave. The polarization angle is defined as the angle between the line of intersection of the gravitational wave plane (the plane transverse to the propagation direction) and the equatorial plane of the earth and the line that is perpendicular to the neutron star's spin direction that also lies in the gravitational wave plane. The signal's frequency at the detector is constantly changing due to spin-down of the neutron star and a Doppler shift caused by the detector's motion relative to the solar system barycentre. This phase evolution information is contained in $\phi(t)$. The signal's amplitude is modulated by the changing orientation of the detector towards the neutron star as the detector rotates with the earth, described by the antenna response $F_{+}(t, \psi)$ and $F_{\times}(t, \psi)$. Equation 1 can therefore be rewritten, expressing the continuous gravitational wave signal in terms of an amplitude $a(t)$ and an oscillatory part $\sin \varphi(t)$ as

$$
\begin{aligned}
h(t)= & \underbrace{h_{0} \sqrt{F_{+}^{2}(t, \psi) \frac{1}{4}\left(1+\cos ^{2} \iota\right)^{2}+F_{\times}^{2}(t, \psi) \cos ^{2} \iota}}_{a(t)} \\
& \times \sin \underbrace{\left[2 \cdot \phi(t)+\arctan \frac{F_{+}(t, \psi)\left(1+\cos ^{2} \iota\right)}{2 \cdot F_{\times}(t, \psi) \cos \iota}\right]}_{\varphi(t)} .
\end{aligned}
$$



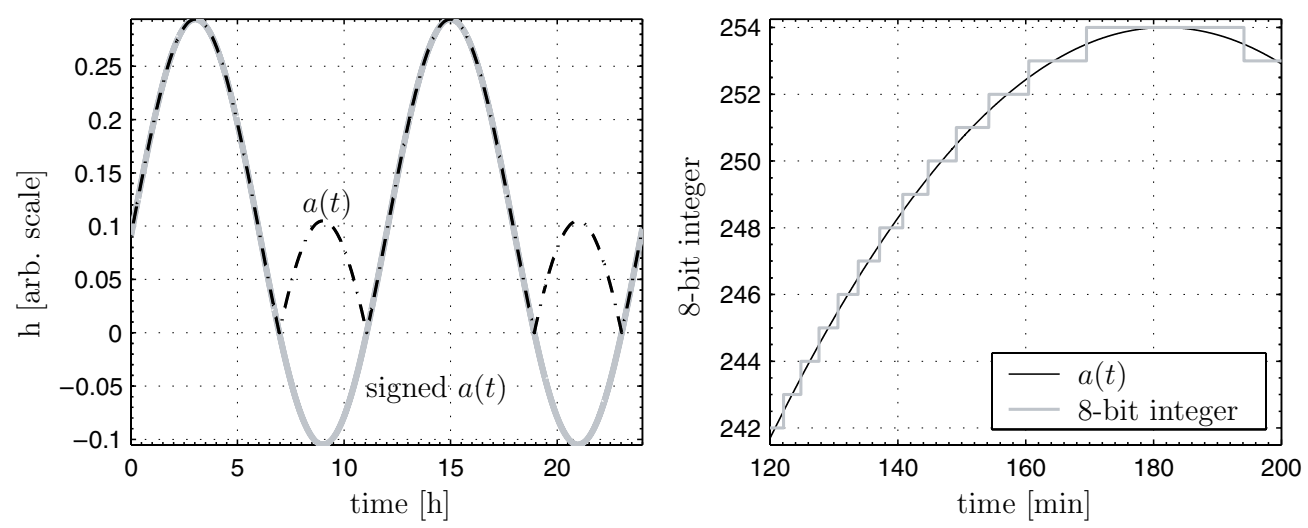

Figure 1. The left-hand graph shows the signal amplitude of a neutron star for the parameters given in section 3 before and after mapping the phase jumps. The right-hand graph shows how the signed signal amplitude $a(t)$ of the left-hand graph is mapped onto an 8-bit D/A converter for minutes 120 to 200 for 2 January 2003.

$h(t), a(t)$ and $\varphi(t)$ can be determined numerically using the LIGO/LSC algorithm library (LAL) [7]. Equation (2) shows that now the amplitude $a(t)$ and the oscillatory part $\sin \varphi(t)$ can be produced electronically independently of each other and then combined by analog electronic multiplication to give the full simulated signal.

\section{Mapping the amplitude}

The amplitude $a(t)$ is defined as a non-negative number so there are jumps of $\pi$ in the phase $\varphi(t)$ that occur at the zeros of the amplitude. As described in section 4 the phase of the signal is controlled in a way that requires a continuous form for $\varphi(t)$, so the amplitude is made bipolar and the phase jumps are converted into a change of the sign of the amplitude of the signal. In summary, the times at which a phase jump occurs are identified and converted into a change of the sign of $a(t)$. This function is then mapped onto an 8-bit D/A converter of the microcontroller which is chosen to be symmetric about zero. As the resulting signal is usually not symmetric about zero, the full dynamic range of the $\mathrm{D} / \mathrm{A}$ converter is exploited only for certain neutron star parameters, depending on which between $60 \%$ to $100 \%$ of the dynamic range of the $\mathrm{D} / \mathrm{A}$ converter is used. The digitization error for a $60 \%$ dynamic range is below $0.7 \%$ and hence better than the amplitude accuracy of the injection hardware [3].

In figure 1 we plot the signal amplitude $a(t)$ of a neutron star scaled to $h_{0}=1$, a signal frequency of $1 \mathrm{kHz}$, right ascension $0^{\circ}$, declination $0^{\circ}, \iota=\pi / 2, \psi=0$ and spin-down of $0 \mathrm{~Hz} \mathrm{~s}^{-1}$ for GEO 600 for 2 January 2003. ${ }^{3}$ In the left-hand graph, the dashed black curve corresponds to $a(t)$ determined with LAL. The grey curve takes phase jumps of $\pi$ into account that occur in $\varphi(t)$, by allowing a negative amplitude. In the right-hand graph the signed signal amplitude of the left-hand graph is mapped onto the 8-bit DA converter for minutes 120 to 200 for 2 January 2003. The signed signal amplitude is mapped into bins 82-254 thus exploiting almost $68 \%$ of the dynamic range. For this signal the time period over which the amplitude is set to the same 8-bit integer is $79 \mathrm{~s}$ at the steepest slope and $1897 \mathrm{~s}$ at the shallowest slope. A sample rate of $1 \mathrm{~Hz}$ was used for these calculations.

3 LAL requires as one input the distance of the source, which was chosen to be 3600 parsec for all simulations in this paper. 


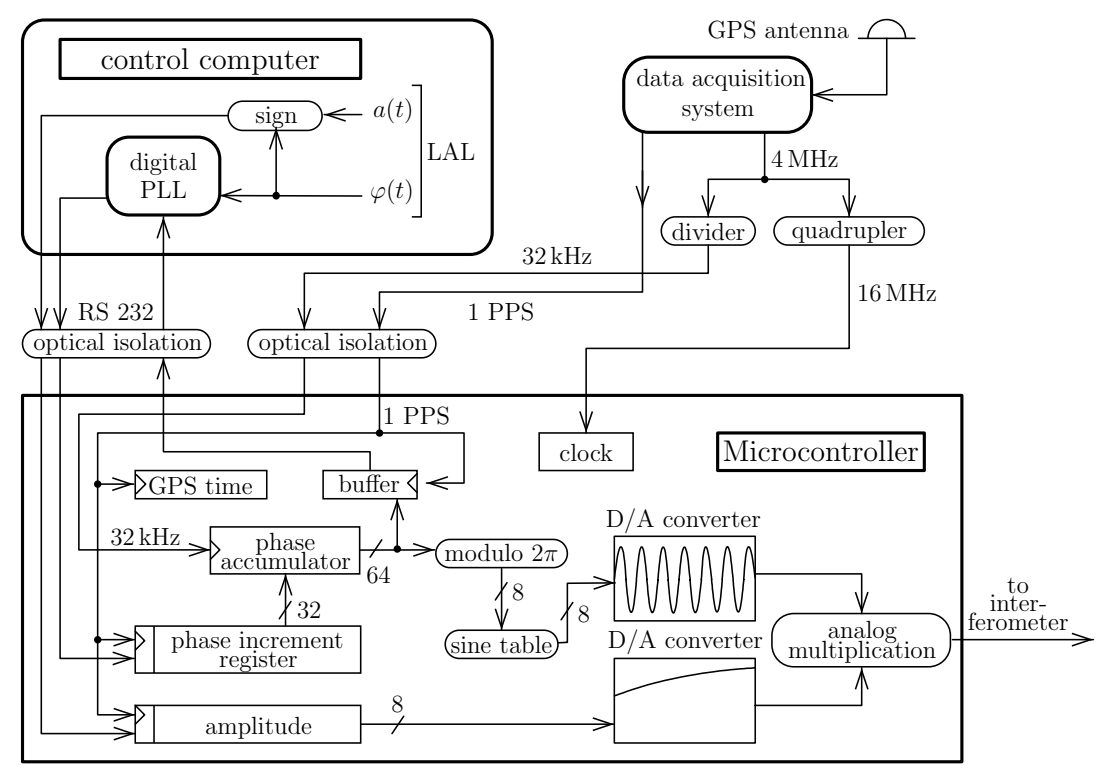

Figure 2. Schematic of the electronic signal production for the hardware injection of continuous gravitational waves. A microcontroller with two D/A converters is used to produce the amplitude $a(t)$ and the oscillatory part of the signal via the phase $\varphi(t)$ of the signal. Before injection into the interferometer they are combined by analog electronic multiplication. The control computer maps the amplitude $a(t)$ onto one of the D/A converters and controls the phase evolution of the signal by a phase-locked loop (PLL).

\section{Controlling the phase}

The main function of the microcontroller is to act as a direct digital frequency synthesizer (DDS). A dedicated DDS was preferred to a commercial unit so that the current value of the phase accumulator could be read out at one-second intervals by the control computer. In addition the size of the phase accumulator was set to 64-bits and is interpreted as a fixed-point number with 32 bits for the integer part (counting multiples of $2 \pi$ ) and 32 bits for the fractional part. A digital phase locked loop (PLL) on the control computer is used to create a signal with the correct phase evolution. The difference between the phase accumulator and the correct signal phase from LAL, $\varphi(t)$, provides the error signal for the control loop. A digital filter is used in the loop to produce a suitable open loop gain, and its output changes the phase increment register in the microcontroller once per second. At a clock frequency of $32 \mathrm{kHz}$, derived from a GPS-locked $4 \mathrm{MHz}$ signal from the GEO 600 data acquisition system [8], the phase increment register is added to the phase accumulator in the microcontroller.

The absolute timing of the signal injection is controlled by pulses of the data acquisition system coinciding with the start of each GPS second. These pulses increment an internal counter of the microcontroller which provides the current GPS second. These pulses are also used as an interrupt to the microcontroller, which initiates one PLL update cycle. An important feature of our system is that the PLL autonomously relocks to the correct signal phase after power on and interruptions once the internal time is synchronized to GPS time. Stable PLLs have been running in a test set-up at Hannover on the final hardware. The phase error in this set-up stayed below $0.3 \%$ of $2 \pi$ with respect to the correct signal phase $\varphi(t)$ over more than a day. Figure 2 shows a schematic diagram of the hardware. 


\section{Conclusion}

We have developed equipment and software for the injection of simulated continuous gravitational waves into a laser interferometric detector, with high phase stability. Accurate timing of the injection process is guaranteed by using GPS-derived clocks and timing pulses. The instrument can autonomously recover from interruptions. In the near future the set-up will be moved to the site of GEO 600 and hardware injection into the interferometer will be investigated and characterized before continuous gravitational wave hardware injection will be employed in scientific data runs.

\section{Acknowledgments}

The authors acknowledge the support of the Deutsche Forschungsgemeinschaft (DFG), Germany and of the Particle Physics and Astronomy Research Council, UK.

\section{References}

[1] Abbott B et al 2004 (for the LIGO Scientific Collaboration) Phys. Rev. D 64 at press (Preprint gr-qc/0308050)

[2] Abbott B et al 2003 (for the LIGO Scientific Collaboration) Detector description and performance for the first coincidence observations between LIGO and GEO Preprint gr-qc/0308043

[3] Hewitson M, Heinzel G, Grote H, Ward H, Strain K and Weiland U 2003 Calibration of the power-recycled GEO 600 Rev. Sci. Instrum. 74 4184-90

[4] Willke B et al 2002 The GEO 600 gravitational wave detector Class. Quantum Grav. 19 1377-87

[5] Goßler S et al 2004 Damping and tuning of the fiber violin modes in monolithic silica suspensions Class. Quantum Grav. 21 S923-33

[6] Jaranowski P, Królak A and Schutz B F 1998 Data analysis of gravitational-wave signals from spinning neutron stars: the signal and its detection Phys. Rev. D 58063001

[7] http://www.lsc-group.phys.uwm.edu/lal/

[8] Koetter K et al 2002 Data acquisition and detector characterisation of GEO 600 Class. Quantum Grav. 19 1399-407 